\title{
Preface to the special issue on inductive logic programming
}

\author{
James Cussens $^{1}$ (D) Alessandra Russo ${ }^{2}$
}

Received: 23 May 2018 / Accepted: 26 May 2018 / Published online: 6 June 2018

(C) The Author(s) 2018

We are pleased to present this special issue of Machine Learning on inductive logic programming (ILP). The papers contained in this volume are extended and thoroughly revised versions of shorter papers which were first presented at the 26th international conference on inductive logic programming (ILP'16).

ILP 2016 was held in London, during September 4-6 2016, at the Warren House Conference Centre. Since its first edition in 1991, the annual ILP conference has served as the premier international forum for learning from structured relational data. Originally focusing on the induction of logic programs, over the years it has expanded its research horizon significantly and welcomed contributions on all aspects of learning in logic, multi-relational data mining, statistical relational learning, graph and tree mining, learning in other (non-propositional) logic-based knowledge representation frameworks, exploring intersections with statistical learning and other probabilistic approaches. Theoretical advances in all these areas have also been accompanied by challenging applications of these techniques to important problems in fields like bioinformatics, medicine, and text mining.

ILP'16 received 35 submissions in total: 10 long papers, 19 short papers, and 6 alreadypublished papers. Of these 35 submissions only 4 out of the unpublished papers have made it into this special issue. Our first paper Meta-Interpretive Learning from noisy images by Muggleton et al. provides a novel approach to image classification called Logical Vision. An approach to ILP called Meta-Interpretative Learning is used to learn an accurate classifier based on as few as a one example, due to the use of rich (learnable) background knowledge. The second paper Ultra-strong machine learning: Comprehensibility of programs learned with ILP, also by Muggleton et al, focuses on what has long been a key 'selling point' of ILP: the comprehensibility (for humans) of what is learned. A key issue addressed by this

James Cussens

James.Cussens@york.ac.uk

Alessandra Russo

A.Russo@imperial.ac.uk

1 University of York, York, UK

2 Imperial College, London, UK 
paper is the impact of predicate invention on human comprehensibility. The third paper Besteffort inductive logic programming via fine-grained cost-based hypothesis generation by Schüller and Benz presents the Inspire system which participated in a competition associated with ILP'16. Inspire generates hypotheses using Answer Set Programming in a way which provides fine-grained control over which hypotheses are in the hypothesis space. The fourth and last paper is Identification of biological transition systems using meta-interpreted logic programs by Srinivasan and Bain. This paper, like the first in this volume, uses a metainterpreter, but here this is used to learn Petri net models of biological systems.

We would like to thank all those involved in making ILP'16 such a success: our invited speakers, our sponsors, the program committee, the conference attendees and of course, the authors whose work we present in this volume. 